\title{
QUANTITATIVE CONCENTRATION INEQUALITIES ON SAMPLE PATH SPACE FOR MEAN FIELD INTERACTION
}

\author{
FRANÇOIS BOLLEY ${ }^{1}$
}

\begin{abstract}
We consider the approximation of a mean field stochastic process by a large interacting particle system. We derive non-asymptotic large deviation bounds measuring the concentration of the empirical measure of the paths of the particles around the law of the process. The method is based on a coupling argument, strong integrability estimates on the paths in Hölder norm, and a general concentration result for the empirical measure of identically distributed independent paths.
\end{abstract}

Mathematics Subject Classification. 82C22, 35K55, 90C08.

Received April 3, 2008. Revised September 29, 2008.

This paper is devoted to the study of the particle approximation of a mean field stochastic process. In the models to be considered, the evolution is governed by a random diffusive term, an exterior force field and a mean field interaction depending on the law of the process itself.

Quantitative estimates on the approximation have been obtained at the level of the time marginals. Let indeed $\mu_{t}$ be the law of the considered process at time $t$, and $\left(X_{t}^{i}\right)_{1 \leq i \leq N}$ be the position of the $N$ particles in the phase space $\mathbb{R}^{d}$; let also $\hat{\mu}_{t}^{N}$ denote their empirical measure. First, [16] adapted concentration of measure ideas to obtain non-asymptotic bounds on the deviation of observables $\frac{1}{N} \sum_{i=1}^{N} \varphi\left(X_{t}^{i}\right)=\int_{\mathbb{R}^{d}} \varphi \mathrm{d} \hat{\mu}_{t}^{N}$ around $\int_{\mathbb{R}^{d}} \varphi \mathrm{d} \mu_{t}$, where $\varphi$ is a given Lipschitz function on $\mathbb{R}^{d}$. Then, transposing Sanov's large deviation argument to their setting, [6] got non-asymptotic bounds on the deviation of $\hat{\mu}_{t}^{N}$ around $\mu_{t}$ at the very level of the measures, namely for a distance which induces a topology stronger that the narrow topology.

In this work we go one step further again by considering the law $\mu_{[0, T]}$ of the paths of the process on a given time interval $[0, T]$. A natural object to consider in the particle approximation is now the empirical measure $\hat{\mu}_{[0, T]}^{N}$ of the $N$ trajectories $\left(X_{t}^{i}\right)_{0 \leq t \leq T}$.

We shall give a precise meaning and estimates on the convergence of $\hat{\mu}_{[0, T]}^{N}$ to $\mu_{[0, T]}$; we shall see that they imply previously mentioned results by projection at time $t$, but above all they give concentration estimates at the level of the paths.

In the first section we state our main results and give an insight of the proofs, which will be given in more detail in the following sections.

\footnotetext{
Keywords and phrases. Mean field limits, particle approximation, transportation inequalities.

${ }^{1}$ Université Paris-Dauphine, Ceremade, UMR 7534, Place du Maréchal de Lattre de Tassigny, 75775 Paris Cedex 16, France; bolley@ceremade.dauphine.fr
} 


\section{Statement of the Results}

We are interested in the particle approximation of the $\mathbb{R}^{d}$-valued process $\left(X_{t}\right)_{t \geq 0}$ evolving according to the mean field stochastic differential equation

$$
\mathrm{d} X_{t}=\sigma \mathrm{d} B_{t}-b\left(X_{t}\right) \mathrm{d} t-c * \mu_{t}\left(X_{t}\right) \mathrm{d} t
$$

Here $\sigma$ is a $d \times d$ real matrix, $\left(B_{t}\right)_{t \geq 0}$ a standard Brownian motion on $\mathbb{R}^{d}, b$ and $c$ are $\mathbb{R}^{d}$ to $\mathbb{R}^{d}$ maps, $*$ stands for the convolution and $\mu_{t}$ is the law on $\mathbb{R}^{d}$ of the random variable $X_{t}$.

Two instances of such processes are particularly interesting. First of all, when $\mathbb{R}^{d}$ is the phase space of positions $x \in \mathbb{R}^{d^{\prime}}$ and velocities $v \in \mathbb{R}^{d^{\prime}}$ with $d=2 d^{\prime}$, one is interested in the process $\left(X_{t}\right)_{t \geq 0}=\left(\left(x_{t}, v_{t}\right)\right)_{t \geq 0}$ solution to the diffusive Newton's equations

$$
\left\{\begin{aligned}
\mathrm{d} x_{t} & =v_{t} \mathrm{~d} t \\
\mathrm{~d} v_{t} & =\sqrt{2} \mathrm{~d} b_{t}-\lambda v_{t} \mathrm{~d} t-\nabla_{x} U *_{x} \rho_{t}\left(x_{t}\right) \mathrm{d} t .
\end{aligned}\right.
$$

Here $\left(b_{t}\right)_{t \geq 0}$ is a Brownian motion in the velocity space $\mathbb{R}^{d^{\prime}}, U=U(x)$ is an interaction potential in the position space and $\rho_{t}$ is the law of $x_{t}$ on $\mathbb{R}^{d^{\prime}}$; moreover $\nabla_{x}$ and $*_{x}$ respectively stand for the gradient and convolution with respect to the position variable $x \in \mathbb{R}^{d^{\prime}}$. By Itô's formula the distribution $\mu_{t}$ of $X_{t}$ is solution to the Vlasov-Fokker-Planck equation

$$
\frac{\partial \mu_{t}}{\partial t}+v \cdot \nabla_{x} \mu_{t}-\left(\nabla_{x} U *_{x} \rho_{t}\right) \cdot \nabla_{v} \mu_{t}=\Delta_{v} \mu_{t}+\lambda \nabla_{v} \cdot\left(v \mu_{t}\right), \quad t>0, x, v \in \mathbb{R}^{d^{\prime}}
$$

Here $a \cdot b$ denotes the scalar product of two vectors $a$ and $b$ in $\mathbb{R}^{d^{\prime}}$, whereas $\nabla_{v}, \nabla_{v} \cdot$ and $\Delta_{v}$ respectively stand for the gradient, divergence and Laplace operators with respect to the velocity variable $v \in \mathbb{R}^{d^{\prime}}$. This equation is used in the modelling of diffusive stellar matter.

We are also concerned with the process $\left(X_{t}\right)_{t \geq 0}=\left(\left(x_{t}, v_{t}\right)\right)_{t \geq 0}$ solution to

$$
\left\{\begin{aligned}
\mathrm{d} x_{t} & =v_{t} \mathrm{~d} t \\
\mathrm{~d} v_{t} & =\sqrt{2} \mathrm{~d} b_{t}-\nabla_{v} V\left(v_{t}\right) \mathrm{d} t-\nabla_{v} W * \nu_{t}\left(v_{t}\right) \mathrm{d} t
\end{aligned}\right.
$$

where $V$ and $W$ are respectively exterior and interaction potentials in the velocity space and $\nu_{t}$ is the law of $v_{t}$ on $\mathbb{R}^{d^{\prime}}$. By Itô's formula, the distribution $\mu_{t}$ of $X_{t}$ is solution to

$$
\frac{\partial \mu_{t}}{\partial t}+v \cdot \nabla_{x} \mu_{t}=\Delta_{v} \mu_{t}+\nabla_{v} \cdot\left(\mu_{t}\left(\nabla_{v} V+\nabla_{v} W *_{v} \mu_{t}\right)\right), \quad t>0, x, v \in \mathbb{R}^{d^{\prime}} .
$$

This equation is used in the modelling of granular media.

For position homogeneous distributions, we are brought to study the solution $\left(v_{t}\right)_{t \geq 0}$ to

$$
\mathrm{d} v_{t}=\sqrt{2} \mathrm{~d} b_{t}-\nabla_{v} V\left(v_{t}\right) \mathrm{d} t-\nabla_{v} W *_{v} \mu_{t}\left(v_{t}\right) \mathrm{d} t
$$

in $\mathbb{R}^{d}$ with $d=d^{\prime}$; here $\mu_{t}$ is the law of $v_{t}$ and is solution to the McKean-Vlasov equation

$$
\frac{\partial \mu_{t}}{\partial t}=\Delta_{v} \mu_{t}+\nabla_{v} \cdot\left(\mu_{t}\left(\nabla_{v} V+\nabla_{v} W *_{v} \mu_{t}\right)\right), \quad t>0, v \in \mathbb{R}^{d^{\prime}}
$$

The particle approximation of such a process consists in introducing $N$ processes $\left(X_{t}^{i}\right)_{t \geq 0}$, with $1 \leq i \leq N$, which evolve no more according to the distribution $\mu_{t}$ of the physical system, but according to its discrete 
counterpart, namely the empirical measure

$$
\hat{\mu}_{t}^{N}=\frac{1}{N} \sum_{i=1}^{N} \delta_{X_{t}^{i}}
$$

of the particle system $\left(X_{t}^{1}, \ldots, X_{t}^{N}\right)$. In other words we let the processes $\left(X_{t}^{i}\right)_{t \geq 0}$ solve

$$
\mathrm{d} X_{t}^{i}=\sigma \mathrm{d} B_{t}^{i}-b\left(X_{t}^{i}\right) \mathrm{d} t-\frac{1}{N} \sum_{j=1}^{N} c\left(X_{t}^{i}-X_{t}^{j}\right) \mathrm{d} t, \quad 1 \leq i \leq N .
$$

Here the $\left(B_{t}^{i}\right)_{t \geq 0}$ 's are $N$ independent standard Brownian motions on $\mathbb{R}^{d}$.

Under regularity and growth assumptions on $\sigma, b$ and $c$, and if the particles are initially distributed in a chaotic way, for instance as independent and identically distributed variables, then $\hat{\mu}_{t}^{N}$ indeed converges as $N$ tends to infinity to the distribution $\mu_{t}$ of $X_{t}$. The convergence of $\hat{\mu}_{t}^{N}$ is strongly linked with the phenomenon of propagation of chaos for the $N$ interacting particles $\left(X_{t}^{i}\right)_{t \geq 0}$, as we shall see below more in detail, and both issues have been studied in $[1,8,17,18]$ for instance. Then quantitative estimates on this convergence have been obtained in [16] at the level of observables, and at the very level of the law in [6].

In this work we go one step further and give precise estimates on the approximation of the law $\mu_{[0, T]}$ of the path $X=\left(X_{t}\right)_{0 \leq t \leq T}$ on a time interval $[0, T]$ by the empirical measure

$$
\hat{\mu}_{[0, T]}^{N}=\frac{1}{N} \sum_{i=1}^{N} \delta_{X^{i}}
$$

of the $N$ trajectories $X^{i}=\left(X_{t}^{i}\right)_{0 \leq t \leq T}$. The convergence of $\hat{\mu}_{[0, T]}^{N}$ has been proved in works mentioned above, and here we extend to this new setting the techniques developed in [6].

We shall assume that $b$ and $c$ are Lipschitz on $\mathbb{R}^{d}$. Then global existence and uniqueness, pathwise and in law, of the solutions to (1.1) and (1.6) are proven in [17] for instance for square integrable initial data; moreover the paths are continuous (in time).

To state our main theorem on the particle approximation, we first give some notation. If $(S, d)$ is a separable and complete metric space, and $p$ is a real number $\geq 1$, the Wasserstein distance of order $p$ between two Borel probability measures $\mu$ and $\nu$ on $S$ is

$$
W_{p}(\mu, \nu):=\inf _{X, Y}\left(\mathbb{E} d(X, Y)^{p}\right)^{1 / p}
$$

where $X$ and $Y$ are $S$-valued random variables with respective law $\mu$ and $\nu$. $W_{p}$ induces a metric on the set of Borel probability measures on $S$ with moment $\int_{S} \mathrm{~d}\left(x_{0}, x\right)^{p} \mathrm{~d} \mu(x)$ finite for some (and thus any) $x_{0}$ in $S$; convergence in this metric is equivalent to narrow convergence plus some tightness condition on the moments (see for instance [20]).

In this work $(S, d)$ will be the space $\mathcal{C}:=\mathcal{C}\left([0, T], \mathbb{R}^{d}\right)$ of $\mathbb{R}^{d}$-valued continuous functions on $[0, T]$, equipped with the uniform norm

for this space $W_{p}$ will be denoted $W_{p,[0, T]}$.

$$
\|f\|_{\infty}:=\sup _{0 \leq t \leq T}|f(t)|
$$

Theorem 1.1. Let $\mu_{0}$ be a probability measure on $\mathbb{R}^{d}$ such that $\int_{\mathbb{R}^{d}} \mathrm{e}^{a_{0}|x|^{2}} \mathrm{~d} \mu_{0}(x)$ be finite for some $a_{0}>0$, and let $b$ and $c$ be Lipschitz functions on $\mathbb{R}^{d}$. Given $T \geq 0$, let $\mu_{[0, T]}$ be the law of the solution to $(1.1)$ on $[0, T]$ 
for some initial value distributed according to $\mu_{0}$. Let also $\left(X_{0}^{i}\right)_{1 \leq i \leq N}$ be $N$ independent random variables with common law $\mu_{0}$ and $\hat{\mu}_{[0, T]}^{N}$ be the empirical measure of the solutions to (1.6) on $[0, T]$, with respective initial value $X_{0}^{i}$.

Then, for any $\alpha \in(0,1 / 2)$, there exist positive constants $K$ and $N_{0}$ such that

$$
\mathbb{P}\left[W_{1,[0, T]}\left(\mu_{[0, T]}, \hat{\mu}_{[0, T]}^{N}\right)>\varepsilon\right] \leq \mathrm{e}^{-K N \varepsilon^{2}}
$$

for all $\varepsilon>0$ and $N \geq N_{0} \varepsilon^{-2} \exp \left(N_{0} \varepsilon^{-1 / \alpha}\right)$.

The constants $K$ and $N_{0}$ depend on $T, b, c, \alpha$ and a finite square exponential moment of $\mu_{0}$.

Kantorovich-Rubinstein dual formulation of the $W_{1}$ distance on a general space $(S, d)$ reads

$$
W_{1}(\mu, \nu)=\sup _{[\varphi]_{1} \leq 1}\left\{\int_{S} \varphi \mathrm{d} \mu-\int_{S} \varphi \mathrm{d} \nu\right\}
$$

where $[\varphi]_{1}:=\sup _{x \neq y} \frac{|\varphi(x)-\varphi(y)|}{\mathrm{d}(x, y)}$. Then the bound in Theorem 1.1 can be written as

$$
\mathbb{P}\left[\sup _{[\varphi]_{1} \leq 1}\left\{\frac{1}{N} \sum_{i=1}^{N} \varphi\left(X^{i}\right)-\int_{\mathcal{C}} \varphi \mathrm{d} \mu_{[0, T]}\right\}>\varepsilon\right] \leq \mathrm{e}^{-K N \varepsilon^{2}}
$$

where the supremum runs over all 1-Lipschitz functions $\varphi$ on $\mathcal{C}$. By projection at time $t$, it implies the concentration inequalities

$$
\mathbb{P}\left[\sup _{[\varphi]_{1} \leq 1}\left\{\frac{1}{N} \sum_{i=1}^{N} \varphi\left(X_{t}^{i}\right)-\int_{\mathbb{R}^{d}} \varphi \mathrm{d} \mu_{t}\right\}>\varepsilon\right] \leq \mathrm{e}^{-\lambda N \varepsilon^{2}}
$$

given in [6] for the time marginals, provided $N \geq N_{0} \varepsilon^{-(d+2)}$ for some $N_{0}$; here the supremum runs over all 1-Lipschitz functions $\varphi$ on $\mathbb{R}^{d}$. But above all it gives error bounds in the approximation by $\frac{1}{N} \sum_{i=1}^{N} \varphi\left(X^{i}\right)$ of the expectation of quantities $\varphi(X)$ which depend on the whole path $X$. In return we impose a stronger condition on the required size of the sample.

An example is the distance $d(X, A)=\inf \left\{\left|X_{t}-y\right| ; t \in[0, T], y \in A\right\}$ of the trajectory to a given set $A$ in $\mathbb{R}^{d}$, which measures how close $X_{t}$ has been to $A$; under the assumptions of Theorem 1.1, for any $\alpha \in(0,1 / 2)$ there exist constants $K$ and $N_{0}$ such that

$$
\mathbb{P}\left[\left|\mathbb{E}[\mathrm{d}(X, A)]-\frac{1}{N} \sum_{i=1}^{N} \mathrm{~d}\left(X^{i}, A\right)\right|>\varepsilon\right] \leq \mathrm{e}^{-K N \varepsilon^{2}}
$$

for any Borel set $A$ in $\mathbb{R}^{d}, \varepsilon>0$ and $N \geq N_{0} \varepsilon^{-2} \exp \left(N_{0} \varepsilon^{-1 / \alpha}\right)$.

A more involved example of such error bounds will be discussed in detail in Section 5 .

As pointed out in [18], the convergence of the empirical measure $\hat{\mu}_{[0, T]}^{N}$ towards the distribution $\mu_{[0, T]}$ is strongly linked with the phenomenon of propagation of chaos, namely, that the interacting particles $X^{i}$ tend to behave like independent variables with law $\mu_{[0, T]}$, as $N$ goes to infinity. For instance, letting

$$
\hat{\mu}_{[0, T]}^{N, 2}:=\frac{1}{N(N-1)} \sum_{i \neq j} \delta_{\left(X^{i}, X^{j}\right)}
$$


be the empirical measure of pairs of paths, the asymptotic independence of two paths (among $N$ ) can be estimated as

Theorem 1.2. With the same notation and assumptions as in Theorem 1.1, for all $T \geq 0$ and $\alpha \in(0,1 / 2)$ there exist positive constants $K$ and $N_{0}$ such that

$$
\mathbb{P}\left[W_{1,[0, T]}\left(\mu_{[0, T]} \otimes \mu_{[0, T]}, \hat{\mu}_{[0, T]}^{N, 2}\right)>\varepsilon\right] \leq \mathrm{e}^{-K N \varepsilon^{2}}
$$

for all $\varepsilon>0$ and $N \geq N_{0} \varepsilon^{-2} \exp \left(N_{0} \varepsilon^{-1 / \alpha}\right)$.

Here the constants $K$ and $N_{0}$ depend on $T, b, c, \alpha$ and a finite square exponential moment of $\mu_{0}$, and $W_{1,[0, T]}$ stands for the Wasserstein distance of order 1 on the product space $\mathcal{C} \times \mathcal{C}$.

In turn this leads to error bounds in the approximation of functions of the paths of two independent solutions to (1.1). Let for instance $\left(X_{t}\right)_{t \geq 0}$ and $\left(\tilde{X}_{t}\right)_{t \geq 0}$ be the solutions to (1.1) respectively driven by two independent Brownian motions $B$ and $\tilde{B}$ and with independent initial data. Then, in average, the minimal distance $\mathrm{d}(X, \tilde{X})=$ $\inf \left\{\left|X_{t}-\tilde{X}_{t}\right| ; t \in[0, T]\right\}$ between $X_{t}$ and $\tilde{X}_{t}$ on $[0, T]$ is approximated by $\frac{1}{N(N-1)} \sum_{i \neq j} d\left(X^{i}, X^{j}\right)$ with an error controlled by

$$
\mathbb{P}\left[\left|\mathbb{E}[\mathrm{d}(X, \tilde{X})]-\frac{1}{N(N-1)} \sum_{i \neq j} \mathrm{~d}\left(X^{i}, X^{j}\right)\right|>\varepsilon\right] \leq \mathrm{e}^{-K N \varepsilon^{2}}
$$

for $\varepsilon>0$ and $N \geq N_{0} \varepsilon^{-2} \exp \left(N_{0} \varepsilon^{-1 / \alpha}\right)$.

The proof of Theorem 1.1 is based on this phenomenon of propagation of chaos: as $N$ goes to infinity, the interacting particles $\left(X_{t}^{i}\right)_{t \geq 0}$ tend to behave like the $N$ independent and identically distributed processes $\left(Y_{t}^{i}\right)_{t \geq 0}$ solution to

$$
\left\{\begin{array}{rlrl}
\mathrm{d} Y_{t}^{i} & =\sigma \mathrm{d} B_{t}^{i}-b\left(Y_{t}^{i}\right) \mathrm{d} t-c * \mu_{t}\left(Y_{t}^{i}\right) \mathrm{d} t & & 1 \leq i \leq N \\
Y_{0}^{i} & =X_{0}^{i}
\end{array}\right.
$$

Here $\mu_{t}$ is the law of $X_{t}$, but is also the law of any $Y_{t}^{i}$ and, for each $i,\left(B_{t}^{i}\right)_{t \geq 0}$ is the Brownian motion driving the evolution of $\left(X_{t}^{i}\right)_{t \geq 0}$. Then the paths $\left(Y_{t}^{i}\right)_{t \geq 0}$ are close to the paths $\left(X_{t}^{i}\right)_{t \geq 0}$ and Proposition 2.1 ensures the existence of a constant $C$ (depending only on $b, c$ and $T$ ) such that

$$
W_{1,[0, T]}\left(\mu_{[0, T]}, \hat{\mu}_{[0, T]}^{N}\right) \leq C W_{1,[0, T]}\left(\mu_{[0, T]}, \hat{\nu}_{[0, T]}^{N}\right)
$$

holds almost surely, where $\hat{\nu}_{[0, T]}^{N}:=\frac{1}{N} \sum_{i=1}^{N} \delta_{Y^{i}}$ and $Y^{i}=\left(Y_{t}^{i}\right)_{0 \leq t \leq T}$; hence controlling the distance between $\mu_{[0, T]}$ and $\hat{\mu}_{[0, T]}^{N}$ reduces to the same issue with $\mu_{[0, T]}$ and $\hat{\nu}_{[0, T]}^{N}$.

But, by definition, the $N$ paths $Y^{i}$ for $1 \leq i \leq N$ are independent and distributed according to $\mu_{[0, T]}$. Then Propositions 3.1 and 4.1 ensure good concentration estimates for the empirical measure $\hat{\nu}_{[0, T]}^{N}$ around the common law $\mu_{[0, T]}$. In the end we obtain the bound

$$
\mathbb{P}\left[W_{1,[0, T]}\left(\mu_{[0, T]}, \hat{\mu}_{[0, T]}^{N}\right)>\varepsilon\right] \leq \mathbb{P}\left[W_{1,[0, T]}\left(\mu_{[0, T]}, \hat{\nu}_{[0, T]}^{N}\right)>\frac{\varepsilon}{C}\right] \leq \mathrm{e}^{-K N \varepsilon^{2}}
$$

under a condition on $\varepsilon$ and $N$.

The proof will be given in greater detail in Section 4 and above all in [4]. It is an adaptation of the argument given in [6], Section 2.6 of estimates (1.8) for time marginals. The current proof turns out to be simpler since it consists in fewer steps; in return each step of the present infinite dimensional case, where $\mu_{[0, T]}$ is a measure on the functional space $\mathcal{C}$, exhibits new difficulties with respect to the finite dimensional case of [6] where $\mu_{t}$ is a measure on $\mathbb{R}^{d}$. 
The proof of Theorem 1.2 consists in writing this coupling argument for pairs of paths and comparing $\mu_{[0, T]} \otimes \mu_{[0, T]}$ and $\hat{\nu}_{[0, T]}^{N, 2}:=\frac{1}{N(N-1)} \sum_{i \neq j} \delta_{\left(Y^{i}, Y^{j}\right)}$ by means of $\frac{1}{N^{2}} \sum_{i, j} \delta_{\left(Y^{i}, Y^{j}\right)}$.

Let us finally note that it would be desirable to relax the assumptions made on the drift terms $b$ and $c$ : first of all to include the interesting case of the cubic potential $W(z)=|z|^{3} / 3$ on $\mathbb{R}$ in the case of equation (1.3), which models the interaction among one-dimensional granular media (see [2] for instance); then to treat the fundamental cases of the Coulomb and gravitational potentials in the diffusive Newton's equations (1.2) (see [11] for instance).

It would also be interesting to consider the whole trajectories $\left(X_{t}\right)_{t \geq 0}$ on $[0,+\infty)$, and derive concentration bounds on functionals such as hitting times for instance.

Before turning to the proofs we briefly give the plan of the paper. In the coming section we reduce our concentration issue on interacting particles to the same issue for independent variables by a coupling argument. In Section 3 we prove a general concentration result for $\mathcal{C}$-valued independent variables and in Section 4 we check that it applies to our framework. An example of error bounds implied by Theorem 1.1 is finally discussed in Section 5.

\section{Coupling}

Let us recall that we want to measure the distance between the law $\mu_{[0, T]}$ of the solution $X=\left(X_{t}\right)_{0 \leq t \leq T}$ on $[0, T]$ to (1.1) and the empirical measure $\hat{\mu}_{[0, T]}^{N}$ of the $N$ solutions $X^{i}=\left(X_{t}^{i}\right)_{0 \leq t \leq T}$ on $[0, T]$ to $(1.6)$.

In the following proposition we reduce this issue to measuring the distance between $\mu_{[0, T]}$ and the empirical measure

$$
\hat{\nu}_{[0, T]}^{N}:=\frac{1}{N} \sum_{i=1}^{N} \delta_{Y^{i}}
$$

of the $N$ independent solutions $Y^{i}=\left(Y_{t}^{i}\right)_{0 \leq t \leq T}$ to $(1.9)$ on $[0, T]$ :

Proposition 2.1. Let us assume that there exist real numbers $\beta, \gamma$ and $\Gamma$ such that

$$
(b(x)-b(y)) \cdot(x-y) \geq \beta|x-y|^{2}, \quad(c(x)-c(y)) \cdot(x-y) \geq \gamma|x-y|^{2}, \quad|c(x)-c(y)| \leq \Gamma|x-y|
$$

for all $x$ and $y$ in $\mathbb{R}^{d}$. Then there exists a constant $C$ depending only on $\beta, \gamma, \Gamma$ and $T$ such that

$$
W_{1,[0, T]}\left(\mu_{[0, T]}, \hat{\mu}_{[0, T]}^{N}\right) \leq C W_{1,[0, T]}\left(\mu_{[0, T]}, \hat{\nu}_{[0, T]}^{N}\right)
$$

almost surely in the above notation.

Proof. We first follow the lines of the proof of [6] or [16] in the case when $b=\nabla V$ and $c=\nabla W$, but in the end we want an estimate on the paths. Since for each $i$ both processes $X^{i}$ and $Y^{i}$ are driven by the same Brownian motion $B^{i}$, the process $X^{i}-Y^{i}$ satisfies

$$
\mathrm{d}\left(X_{t}^{i}-Y_{t}^{i}\right)=-\left(b\left(X_{t}^{i}\right)-b\left(Y_{t}^{i}\right)\right) \mathrm{d} t-\left(c * \hat{\mu}_{t}^{N}\left(X_{t}^{i}\right)-c * \mu_{t}\left(Y_{t}^{i}\right)\right) \mathrm{d} t .
$$

In particular

$$
\frac{1}{2} \frac{\mathrm{d}}{\mathrm{d} t}\left|X_{t}^{i}-Y_{t}^{i}\right|^{2}=-\left(b\left(X_{t}^{i}\right)-b\left(Y_{t}^{i}\right)\right) \cdot\left(X_{t}^{i}-Y_{t}^{i}\right)-\left(c * \hat{\mu}_{t}^{N}\left(X_{t}^{i}\right)-c * \mu_{t}\left(Y_{t}^{i}\right)\right) \cdot\left(X_{t}^{i}-Y_{t}^{i}\right) .
$$

We decompose the last term according to

$$
c * \hat{\mu}_{t}^{N}\left(X_{t}^{i}\right)-c * \mu_{t}\left(Y_{t}^{i}\right)=\left(c * \hat{\mu}_{t}^{N}-c * \mu_{t}\right)\left(X_{t}^{i}\right)+\left(c * \mu_{t}\left(X_{t}^{i}\right)-c * \mu_{t}\left(Y_{t}^{i}\right)\right) .
$$


Then the map $c\left(X_{t}^{i}-\cdot\right)$ is $\Gamma$-Lipschitz, so the Kantorovich-Rubinstein formulation (1.7) of the Wasserstein distance of order 1 ensures that

$$
\left|c *\left(\hat{\mu}_{t}^{N}-\mu_{t}\right)\left(X_{t}^{i}\right)\right|=\left|\int_{\mathbb{R}^{d}} c\left(X_{t}^{i}-y\right) \mathrm{d}\left(\hat{\mu}_{t}^{N}-\mu_{t}\right)(y)\right| \leq \Gamma w_{1}\left(\hat{\mu}_{t}^{N}, \mu_{t}\right)
$$

where $w_{p}$ is the Wasserstein distance of order $p \geq 1$ between measures on $\mathbb{R}^{d}$ equipped with the Euclidean distance $|\cdot|$. Then (2.2) and the assumptions on $b$ and $c$ imply

$$
\frac{1}{2} \frac{\mathrm{d}}{\mathrm{d} t}\left|X_{t}^{i}-Y_{t}^{i}\right|^{2} \leq \Gamma w_{1}\left(\hat{\mu}_{t}^{N}, \mu_{t}\right)\left|X_{t}^{i}-Y_{t}^{i}\right|-(\beta+\gamma)\left|X_{t}^{i}-Y_{t}^{i}\right|^{2} .
$$

In particular, by integration,

$$
\left|X_{s}^{i}-Y_{s}^{i}\right| \leq \Gamma \int_{0}^{s} w_{1}\left(\hat{\mu}_{u}^{N}, \mu_{u}\right) \mathrm{d} u-(\beta+\gamma) \int_{0}^{s}\left|X_{u}^{i}-Y_{u}^{i}\right| \mathrm{d} u
$$

since initially $X_{0}^{i}=Y_{0}^{i}$. But

$$
w_{1}\left(\hat{\mu}_{u}^{N}, \mu_{u}\right) \leq W_{1,[0, u]}\left(\hat{\mu}_{[0, u]}^{N}, \mu_{[0, u]}\right)
$$

since $\hat{\mu}_{u}^{N}$ and $\mu_{u}$ are the respective image measures of $\hat{\mu}_{[0, u]}^{N}$ and $\mu_{[0, u]}$ by the 1-Lipschitz projection $\pi_{u}$ from $\mathcal{C}\left([0, u], \mathbb{R}^{d}\right)$ into $\mathbb{R}^{d}$ defined by $\pi_{u}(f)=f(u)$. Moreover

$$
W_{1,[0, u]}\left(\hat{\mu}_{[0, u]}^{N}, \mu_{[0, u]}\right) \leq \frac{1}{N} \sum_{i=1}^{N} \sup _{0 \leq s \leq u}\left|X_{s}^{i}-Y_{s}^{i}\right|+W_{1,[0, u]}\left(\hat{\nu}_{[0, u]}^{N}, \mu_{[0, u]}\right) .
$$

by triangular inequality. Hence, by averaging (2.4) over $i$, and by Gronwall's lemma,

$$
\frac{1}{N} \sum_{i=1}^{N} \sup _{0 \leq s \leq t}\left|X_{s}^{i}-Y_{s}^{i}\right| \leq \Gamma \int_{0}^{t} \mathrm{e}^{(\Gamma+m)(t-u)} W_{1,[0, u]}\left(\hat{\nu}_{[0, u]}^{N}, \mu_{[0, u]}\right) \mathrm{d} u
$$

where $m:=\max (0,-(\beta+\gamma))$. On the other hand, for $0 \leq u \leq t$,

$$
W_{1,[0, u]}\left(\hat{\nu}_{[0, u]}^{N}, \mu_{[0, u]}\right) \leq W_{1,[0, t]}\left(\hat{\nu}_{[0, t]}^{N}, \mu_{[0, t]}\right)
$$

since $\hat{\nu}_{[0, u]}^{N}$ and $\mu_{[0, u]}$ are the respective image measures of $\hat{\nu}_{[0, t]}^{N}$ and $\mu_{[0, t]}$ by the 1-Lipschitz map defined from $\mathcal{C}\left([0, t], \mathbb{R}^{d}\right)$ into $\mathcal{C}\left([0, u], \mathbb{R}^{d}\right)$ as the restriction to $[0, u]$. Hence

$$
W_{1,[0, t]}\left(\hat{\mu}_{[0, t]}^{N}, \hat{\nu}_{[0, t]}^{N}\right) \leq \frac{1}{N} \sum_{i=1}^{N} \sup _{0 \leq s \leq t}\left|X_{s}^{i}-Y_{s}^{i}\right| \leq \Gamma(\Gamma+m)^{-1}\left(\mathrm{e}^{(\Gamma+m) T}-1\right) W_{1,[0, t]}\left(\hat{\nu}_{[0, t]}^{N}, \mu_{[0, t]}\right)
$$

by (2.5). This concludes the argument by triangular inequality.

Remark 2.1. In the case of the granular media equation (1.4), and under convexity assumptions on $V$ and $W$, such as $\beta>0, \beta+2 \gamma>0$, it has been proven in $[7,8,16]$ that the time marginal $\mu_{t}$ converges, as $t$ goes to infinity, to the stationary solution to (1.5); one can also prove that in expectation observables of the particle system are bounded in time.

Hence, under this kind of assumptions, one can hope for uniform in time constants in this coupling argument. This was obtained in [6] for the time marginals of the granular media equation; here, for the whole processes, 
if $\beta+\gamma>\Gamma$, then we can let $C$ be $(\beta+\gamma)(\beta+\gamma-\Gamma)^{-1}$ in Proposition 2.1, independently of $T$. Indeed, if $\beta+\gamma>0$, then (2.3) leads to

$$
\left|X_{s}^{i}-Y_{s}^{i}\right| \leq \Gamma \int_{0}^{s} \mathrm{e}^{-(\beta+\gamma)(s-u)} w_{1}\left(\hat{\mu}_{u}^{N}, \mu_{u}\right) \mathrm{d} u
$$

by integration. Consequently

$$
W_{1,[0, t]}\left(\hat{\mu}_{[0, t]}^{N}, \hat{\nu}_{[0, t]}^{N}\right) \leq \Gamma \sup _{0 \leq s \leq t} \int_{0}^{s} \mathrm{e}^{-(\beta+\gamma)(s-u)} \mathrm{d} u \sup _{0 \leq u \leq t} w_{1}\left(\hat{\mu}_{u}^{N}, \mu_{u}\right) \leq \frac{\Gamma}{\beta+\gamma} W_{1,[0, t]}\left(\hat{\mu}_{[0, t]}^{N}, \mu_{[0, t]}\right) .
$$

If moreover $\beta+\gamma>\Gamma$, then by triangular inequality

$$
W_{1,[0, t]}\left(\hat{\mu}_{[0, t]}^{N}, \mu_{[0, t]}\right) \leq \frac{\beta+\gamma}{\beta+\gamma-\Gamma} W_{1,[0, t]}\left(\hat{\nu}_{[0, t]}^{N}, \mu_{[0, t]}\right) .
$$

\section{A PRELIMINARY RESUlt ON INDEPENDENT VARIABLES}

Our main theorem on the particle approximation is based on a general concentration result for the empirical measure of $\mathcal{C}$-valued independent and identically distributed random variables. In this section we state this result in a more general formulation and for this purpose we first introduce some notation.

If $\mu$ and $\nu$ are two measures on $\mathcal{C}$, the relative entropy of $\nu$ with respect to $\mu$ is defined by

$$
H(\nu \mid \mu)=\int_{C} \frac{\mathrm{d} \nu}{\mathrm{d} \mu} \ln \frac{\mathrm{d} \nu}{\mathrm{d} \mu} \mathrm{d} \mu
$$

if $\nu$ is absolutely continuous with respect to $\mu$, and $H(\nu \mid \mu)=+\infty$ otherwise.

This notion is linked with the Wasserstein distances by the family of transportation inequalities: given $p \geq 1$ and $\lambda>0$, a probability measure $\mu$ on $C$ satisfies the inequality $T_{p}(\lambda)$ if

$$
W_{p}(\mu, \nu) \leq \sqrt{\frac{2}{\lambda} H(\nu \mid \mu)}
$$

holds true for any measure $\nu$.

Moreover, given a Borel probability measure $\mu$ on $\mathcal{C}$ and $N$ independent random variables $\left(X^{i}\right)_{1 \leq i \leq N}$ with law $\mu$, we let $\hat{\mu}^{N}$ denote their empirical measure, defined as

$$
\hat{\mu}^{N}:=\frac{1}{N} \sum_{i=1}^{N} \delta_{X^{i}} .
$$

Given a real number $\alpha \in(0,1]$, we let $\mathcal{C}^{\alpha}:=\mathcal{C}^{\alpha}\left([0, T], \mathbb{R}^{d}\right)$ be the space of functions in $\mathcal{C}$ which moreover are Hölder of order $\alpha$, equipped with the Hölder norm

$$
\|f\|_{\alpha}:=\sup \left(\|f\|_{\infty},[f]_{\alpha}\right)
$$

where

$$
[f]_{\alpha}:=\sup _{0 \leq t, s \leq T} \frac{|f(t)-f(s)|}{|t-s|^{\alpha}} .
$$


$\mathcal{C}^{\alpha}$ is a Borel set of the space $\mathcal{C}$ equipped with the topology induced by the uniform norm, and for Borel measures on $\mathcal{C}$, concentrated on $\mathcal{C}^{\alpha}$, we have in the above notation:

Proposition 3.1. Let $\mu$ be a Borel probability measure on $\mathcal{C}$ satisfying a $T_{p}(\lambda)$ inequality for some $\lambda>0$ and $p \in[1,2]$, and such that $\int_{\mathcal{C}} \mathrm{e}^{a\|x\|_{\alpha}^{2}} \mathrm{~d} \mu(x)$ be finite for some $a>0$ and $\alpha \in(0,1]$. Then, for any $\alpha^{\prime}<\alpha$ and $\lambda^{\prime}<\lambda$, there exists a constant $N_{0}$ such that

$$
\mathbb{P}\left[W_{p,[0, T]}\left(\mu, \hat{\mu}^{N}\right)>\varepsilon\right] \leq \mathrm{e}^{-\beta_{p} \frac{\lambda^{\prime}}{2} N \varepsilon^{2}}
$$

for any $\varepsilon>0$ and $N \geq N_{0} \varepsilon^{-2} \exp \left(N_{0} \varepsilon^{-1 / \alpha^{\prime}}\right)$, where

$$
\beta_{p}=\left\{\begin{array}{ccc}
1 & \text { if } & 1 \leq p<2 \\
(1+\sqrt{\lambda / a})^{-2} & \text { if } & p=2
\end{array}\right.
$$

Here the constant $N_{0}$ depends on $\mu$ only through $\lambda, a, \alpha$ and $\int_{\mathcal{C}} \mathrm{e}^{a\|x\|_{\alpha}^{2}} \mathrm{~d} \mu(x)$.

This proposition will be applied with $p=1$ to the distribution $\mu_{[0, T]}$ of the process $X$. For $p=1$, a $T_{1}(\lambda)$ inequality is equivalent to the existence of $a>0$ such that $\int_{\mathcal{C}} \mathrm{e}^{a\|x\|_{\infty}^{2}} \mathrm{~d} \mu(x)$ be finite (see [10]). Numerical relations between such $a$ and $\lambda$ are given in [5,13]. In particular this condition if fulfilled if $\int_{\mathcal{C}} \mathrm{e}^{a\|x\|_{\alpha}^{2}} \mathrm{~d} \mu(x)$ is finite, as will be the case for $\mu_{[0, T]}$.

For $p=1$ again, a result by Bobkov and Götze [3], based on (1.7), ensures that a $T_{1}(\lambda)$ inequality for $\mu$ is equivalent to the following precise version of the central limit theorem:

$$
\sup _{[\varphi]_{1} \leq 1} \mathbb{P}\left[\frac{1}{N} \sum_{i=1}^{N} \varphi\left(X^{i}\right)-\int_{C} \varphi \mathrm{d} \mu>\varepsilon\right] \leq \mathrm{e}^{-\frac{\lambda}{2} N \varepsilon^{2}}, \quad N \geq 1 .
$$

By comparison, the bound given in Proposition 3.1 implies

$$
\mathbb{P}\left[\sup _{[\varphi]_{1} \leq 1}\left\{\frac{1}{N} \sum_{i=1}^{N} \varphi\left(X^{i}\right)-\int_{\mathcal{C}} \varphi \mathrm{d} \mu\right\}>\varepsilon\right] \leq \mathrm{e}^{-\frac{\lambda^{\prime}}{2} N \varepsilon^{2}}, \quad \lambda^{\prime}<\lambda, \quad N \text { large enough }
$$

by (1.7), but a modification of the proof would also lead to

$$
\mathbb{P}\left[\sup _{[\varphi]_{1} \leq 1}\left\{\frac{1}{N} \sum_{i=1}^{N} \varphi\left(X^{i}\right)-\int_{\mathcal{C}} \varphi \mathrm{d} \mu\right\}>\varepsilon\right] \leq C(\varepsilon) \mathrm{e}^{-\frac{\lambda^{\prime}}{2} N \varepsilon^{2}}, \quad \lambda^{\prime}<\lambda, \quad N \geq 1
$$

for some computable large constant $C(\varepsilon)$. Thus we control a much stronger quantity, up to some loss on the constant in the right-hand side or some condition on the sample size.

This result is reasonable in view of Sanov's theorem (stated in [9] for instance). Indeed, by this theorem, one can hope for an upper bound like

$$
\mathbb{P}\left[W_{p,[0, T]}\left(\mu, \hat{\mu}^{N}\right)>\varepsilon\right] \leq \exp (-N \inf \{H(\nu \mid \mu) ; \nu \in A\})
$$

for large $N$, where $A:=\left\{\nu ; W_{p,[0, T]}(\nu, \mu)>\varepsilon\right\}$. But $\mu$ satisfies $T_{p}(\lambda)$, so that

$$
\inf \{H(\nu \mid \mu) ; \nu \in A\} \geq \frac{\lambda}{2} \varepsilon^{2},
$$


and one indeed obtains an upper bound like (3.1), but only asymptotically, whereas Proposition 3.1 gives a sufficient size of the sample for the deviation bound to hold. Sanov's theorem does not actually give such an upper bound here: indeed, the space $\mathcal{C}$ being unbounded, the closure $\bar{A}$ of $A$ (for the narrow topology) contains $\mu$ itself; in particular $\inf \{H(\nu \mid \mu) ; \nu \in \bar{A}\}=0$ and Sanov's theorem only gives the trivial bound $\mathbb{P}\left[\hat{\mu}^{N} \in A\right] \leq \exp (-N \inf \{H(\nu \mid \mu) ; \nu \in \bar{A}\})=1$.

Finally this result can be seen as an extension of the following similar concentration result given in [6], Theorem 2.1 in the case of measures on $\mathbb{R}^{d}$ : if $m$ satisfies $T_{p}(\lambda)$, then

$$
\mathbb{P}\left[w_{p}\left(m, \hat{m}^{N}\right)>\varepsilon\right] \leq \mathrm{e}^{-\gamma_{p} \frac{\lambda^{\prime}}{2} N \varepsilon^{2}}, \quad \varepsilon>0, \quad N \geq N_{0} \max \left(\varepsilon^{-\left(d^{\prime}+2\right)}, 1\right) .
$$

Let indeed $m$ be such a measure on $\mathbb{R}^{d}$. Then the law $\mu$ of a constant process on $[0, T]$ initially distributed according to $m$ satisfies the assumptions of Proposition 3.1 (one can take any $a<\lambda / 2$ ), and the bound (3.2) follows by (3.1) with the constant $\gamma_{p}$ obtained in [6]. Note however that the required size of the sample is here larger for small $\varepsilon$.

Proof. The proof goes in four steps: we first reduce the problem to a ball $\mathcal{B}_{R}^{\alpha}$ of $\mathcal{C}^{\alpha}$, compact in $\mathcal{C}$, which has almost full $\mu$ measure by the integrability assumption on $\mu$; then we cover $\mathcal{B}_{R}^{\alpha}$ and then $\mathcal{P}\left(\mathcal{B}_{R}^{\alpha}\right)$ by small balls on which we develop Sanov's argument as in [14] and [9], exercises 4.4.5 and 6.2.19; we estimate the cardinality of these covering balls; then we conclude the proof by optimizing the introduced parameters. The argument has the same plan as in the finite dimensional case of [6], Section 3.1, so we shall only sketch it, stressing only on the bounds specific to the present infinite dimensional case. We refer to [6] for further details.

\subsection{Truncation}

Given $R>0$, to be chosen later on, we let $\mathcal{B}_{R}^{\alpha}$ denote the ball $\left\{f \in \mathcal{C}^{\alpha} ;\|f\|_{\alpha} \leq R\right\}$ of center 0 and radius $R$ in $\mathcal{C}^{\alpha}$. This set $\mathcal{B}_{R}^{\alpha}$ is a compact subset of $\mathcal{C}$ for the topology induced by the uniform norm $\|\cdot\|_{\infty}$. Letting $\mathbf{1}_{\mathcal{B}_{R}^{\alpha}}$ be the indicator function of $\mathcal{B}_{R}^{\alpha}$, we truncate $\mu$ into a probability measure $\mu_{R}$ on the ball $\mathcal{B}_{R}^{\alpha}$, defined as

$$
\mu_{R}:=\mathbf{1}_{\mathcal{B}_{R}^{\alpha}} \mu / \mu\left[\mathcal{B}_{R}^{\alpha}\right] .
$$

Note that $\mu\left[\mathcal{B}_{R}^{\alpha}\right]$ is positive for $R$ larger than some $R_{0}$ depending only on $E:=\int_{\mathcal{C}} \mathrm{e}^{a\|x\|_{\alpha}^{2}} \mathrm{~d} \mu(x)$ and $a$. In this step we reduce the concentration issue for $\mathcal{C}$ to the same issue for the compact ball $\mathcal{B}_{R}^{\alpha}$, by bounding the quantity $\mathbb{P}\left[W_{p,[0, T]}\left(\mu, \hat{\mu}^{N}\right)>\varepsilon\right]$ in terms of $\mu_{R}$ and an associated empirical measure $\hat{\mu}_{R}^{N}:=\frac{1}{N} \sum_{k=1}^{N} \delta_{X_{R}^{k}}$ where the $X_{R}^{k}$ are independent variables with law $\mu_{R}$.

Bounding by above the $\|\cdot\|_{\infty}$ norms by $\|\cdot\|_{\alpha}$ norms when necessary, we obtain the bound

$$
\begin{aligned}
\mathbb{P}\left[W_{p,[0, T]}\left(\mu, \hat{\mu}^{N}\right)>\varepsilon\right] \leq \mathbb{P}\left[W_{p,[0, T]}\left(\mu_{R}, \hat{\mu}_{R}^{N}\right)>\eta \varepsilon-2 E^{\frac{1}{p}} R \mathrm{e}^{-\frac{a}{p} R^{2}}\right] & \\
& +\exp \left(-N\left(\theta(1-\eta)^{p} \varepsilon^{p}-E \mathrm{e}^{\left(a_{1}-a\right) R^{2}}\right)\right) ;
\end{aligned}
$$

here $p$ is any real number in $[1,2), \eta$ in $(0,1), \varepsilon, \theta>0, a_{1}<a$ and $R$ is constrained to be larger than $R_{2} \max \left(1, \theta^{\frac{1}{2-p}}\right)$ for some constant $R_{2}$ depending only on $E, a, a_{1}$ and $p$.

In the case when $p=2$, we obtain

$$
\begin{aligned}
\mathbb{P}\left[W_{2,[0, T]}\left(\mu, \hat{\mu}^{N}\right)>\varepsilon\right] \leq \mathbb{P}\left[W_{2,[0, T]}\left(\mu_{R}, \hat{\mu}_{R}^{N}\right)>\eta \varepsilon-2 E^{\frac{1}{2}} R \mathrm{e}^{-\frac{a}{2} R^{2}}\right] \\
+\exp \left(-N\left(\frac{a_{1}}{2}(1-\eta)^{2} \varepsilon^{2}-2 E^{2} \mathrm{e}^{\left(a_{1}-a\right) R^{2}}\right)\right) .
\end{aligned}
$$




\subsection{Sanov's argument on small balls}

In view of (3.3) for $p<2$ or (3.4) for $p=2$, we now aim at bounding $\mathbb{P}\left[\hat{\mu}_{R}^{N} \in \mathcal{A}\right]$ where

$$
\mathcal{A}:=\left\{\nu \in \mathcal{P}\left(\mathcal{B}_{R}^{\alpha}\right) ; \quad W_{p,[0, T]}\left(\mu_{R}, \nu\right) \geq \eta \varepsilon-2 E^{\frac{1}{p}} R \mathrm{e}^{-\frac{a}{p} R^{2}}\right\} .
$$

For this we let $\delta>0$ and cover $\mathcal{A}$ with $\mathcal{N}_{p}(\mathcal{A}, \delta)$ balls $B_{i}$ with radius $\delta / 2$ in $W_{p,[0, T]}$ distance. Then we develop Sanov's argument on each of these compact and convex balls to obtain

$$
\mathbb{P}\left[\hat{\mu}_{R}^{N} \in \mathcal{A}\right] \leq \mathbb{P}\left[\hat{\mu}_{R}^{N} \in \bigcup_{i=1}^{\mathcal{N}_{p}(\mathcal{A}, \delta)} B_{i}\right] \leq \sum_{i=1}^{\mathcal{N}_{p}(\mathcal{A}, \delta)} \mathbb{P}\left[\hat{\mu}_{R}^{N} \in B_{i}\right] \leq \sum_{i=1}^{\mathcal{N}_{p}(\mathcal{A}, \delta)} \exp \left(-N \inf _{\nu \in B_{i}} H\left(\nu \mid \mu_{R}\right)\right) .
$$

Then, from the $T_{p}(\lambda)$ inequality for $\mu$, one establishes an approximate $T_{p}(\lambda)$ inequality for $\mu_{R}$ : namely, for any $\lambda_{1}<\lambda$ there exists $K_{1}$ such that

$$
H\left(\nu, \mu_{R}\right) \geq \frac{\lambda_{1}}{2} W_{p,[0, T]}\left(\nu, \mu_{R}\right)^{2}-K_{1} R^{2} \mathrm{e}^{-a R^{2}}
$$

for any measure $\nu$ on $\mathcal{B}_{R}^{\alpha}$. With this inequality in hand, given $1 \leq p<2$ and $\lambda_{2}<\lambda_{1}<\lambda$, one deduces from (3.5) the existence of positive constants $\delta_{1}, \eta_{1}$ and $K_{1}$ such that

$$
\mathbb{P}\left[W_{p,[0, T]}\left(\mu_{R}, \hat{\mu}_{R}^{N}\right)>\eta \varepsilon-2 E^{\frac{1}{p}} R \mathrm{e}^{-\frac{a}{p} R^{2}}\right] \leq \mathcal{N}_{p}(\mathcal{A}, \delta) \exp \left(-N\left(\frac{\lambda_{2}}{2} \varepsilon^{2}-K_{1} R^{2} \mathrm{e}^{-a R^{2}}\right)\right)
$$

where we have chosen $\delta:=\delta_{1} \varepsilon$ and $\eta:=\eta_{1}$.

In the case when $p=2$, we do not choose $\eta$ at this stage, and simply obtain

$$
\mathbb{P}\left[W_{2,[T]}\left(\mu_{R}, \hat{\mu}_{R}^{N}\right)>\eta \varepsilon-2 E^{\frac{1}{2}} R \mathrm{e}^{-\frac{a}{2} R^{2}}\right] \leq \mathcal{N}_{p}(\mathcal{A}, \delta) \exp \left(-N\left(\frac{\lambda_{2}}{2} \eta^{2} \varepsilon^{2}-K_{1} R^{2} \mathrm{e}^{-a R^{2}}\right)\right)
$$

where $\delta:=\delta_{1} \varepsilon$.

\subsection{Estimate on the covering number $\mathcal{N}_{p}(\mathcal{A}, \delta)$}

Given $r>0$, the covering number $\mathcal{N}(S, r)$ of a compact metric space $(S, d)$ is the smallest integer $n$ such that $S$ can be covered by $n$ balls centered in $S$ and of radius $r$ in $d$ metric. On $\mathcal{N}\left(\mathcal{B}_{R}^{\alpha}, r\right)$ we have the following bound:

Lemma 3.1. Given the space $\mathbb{R}^{d}$ with $d \geq 1$, some positive numbers $T, R, r$ and $\alpha$ with $r<R$ and $\alpha \leq 1$, the covering number $\mathcal{N}\left(\mathcal{B}_{R}^{\alpha}, r\right)$ of $\mathcal{B}_{R}^{\alpha}$, equipped with the uniform norm, satisfies

$$
\mathcal{N}\left(\mathcal{B}_{R}^{\alpha}, r\right) \leq\left(10 \sqrt{d} \frac{R}{r}\right)^{d} 3^{5^{\frac{1}{\alpha}} d^{1+\frac{1}{2 \alpha}} T\left(\frac{R}{r}\right)^{\frac{1}{\alpha}}} .
$$

The proof and a lower bound of the same order are given in detail in [4]; in our case these bounds make more precise those given in $[13,15]$ or $[19]$.

Moreover, given a compact metric space $(S, d), p \geq 1$ and $\delta>0$, the following result of [6] (see also [9,14]) gives an upper bound on the covering number $\mathcal{N}_{p}(\mathcal{P}(S), \delta)$ of the compact metric space $\left(\mathcal{P}(S), W_{p}\right)$ of probability measures on $S$ :

Lemma 3.2. Let $(S, d)$ be a compact metric space with finite diameter $D$, and $p$ and $\delta$ be positive numbers with $p \geq 1$. Then the covering numbers of $S$ and $\mathcal{P}(S)$ satisfy

$$
\begin{array}{lll}
\mathcal{N}_{p}(\mathcal{P}(S), \delta) \leq\left(8 \mathrm{e} \frac{D}{\delta}\right)^{p \mathcal{N}\left(S, \frac{\delta}{2}\right)} & & \text { if } 0<\delta<D \\
\mathcal{N}_{p}(\mathcal{P}(S), \delta) & =1 & \text { if } \delta \geq D .
\end{array}
$$


Since $\mathcal{B}_{R}^{\alpha}$ equipped with the metric defined by the uniform norm is a compact metric space with finite diameter $2 R$, it follows that the space $\mathcal{P}\left(\mathcal{B}_{R}^{\alpha}\right)$ of probability measures on $\mathcal{B}_{R}^{\alpha}$ can be covered by $\mathcal{N}_{p}\left(\mathcal{P}\left(\mathcal{B}_{R}^{\alpha}\right), \delta\right)$ balls of radius $\delta$ in Wasserstein distance $W_{p}$, with

$$
\begin{array}{ll}
\mathcal{N}_{p}\left(\mathcal{P}\left(\mathcal{B}_{R}^{\alpha}\right), \delta\right) \leq\left(16 e R \delta^{-1}\right)^{p\left(20 \sqrt{d} R \delta^{-1}\right)^{d} 3^{10^{\frac{1}{\alpha}} d^{1+\frac{1}{2 \delta}} T\left(R \delta^{-1}\right)^{\frac{1}{\alpha}}}} & \text { if } 0<\delta<2 R \\
\mathcal{N}_{p}\left(\mathcal{P}\left(\mathcal{B}_{R}^{\alpha}\right), \delta\right)=1 & \text { if } \delta \geq 2 R .
\end{array}
$$

Then $\mathcal{A}$ is a subset of $\mathcal{P}\left(\mathcal{B}_{R}^{\alpha}\right)$, so, for a constant $K_{2}$ depending neither on $\varepsilon$ nor on $R$,

$$
\mathcal{N}_{p}\left(\mathcal{A}, \delta_{1} \varepsilon\right) \leq \exp \left(K_{2}\left(R \varepsilon^{-1}\right)^{d} 3^{K_{2}\left(R \varepsilon^{-1}\right)^{1 / \alpha}} \ln \left(\max \left(1, K_{2} R \varepsilon^{-1}\right)\right)\right) .
$$

Remark 3.1. The order of magnitude of this covering number in an infinite dimensional setting constitutes a main change by comparison with the finite dimensional setting of [6], and will influence the final condition on the size $N$ of the sample.

\subsection{Conclusion of the argument}

We first focus on the case when $p \in[1,2)$. By estimates (3.3), (3.6) and (3.7), and given $\lambda_{2}<\lambda$ and $a_{1}<a$, we obtain the existence of positive constants $K_{1}, K_{2}, K_{3}$ and $R_{3}$ depending on $E, a, a_{1}, \alpha, \lambda$ and $\lambda_{2}$ such that

$$
\begin{aligned}
\mathbb{P}\left[W_{p,[0, T]}\left(\mu, \hat{\mu}^{N}\right)>\right. & \varepsilon] \leq \exp \left(-N\left(K_{3} \theta \varepsilon^{p}-K_{4} \mathrm{e}^{\left(a_{1}-a\right) R^{2}}\right)\right) \\
& +\exp \left(K_{2}\left(R \varepsilon^{-1}\right)^{d} 3^{K_{2}\left(R \varepsilon^{-1}\right)^{1 / \alpha}} \ln \left(\max \left(1, K_{2} R \varepsilon^{-1}\right)\right)-N\left(\frac{\lambda_{2}}{2} \varepsilon^{2}-K_{1} R^{2} \mathrm{e}^{-\alpha R^{2}}\right)\right)
\end{aligned}
$$

for all $\varepsilon, \theta>0$ and $R \geq R_{3} \max \left(1, \theta^{\frac{1}{2-p}}\right)$, and for a constant $K_{4}=K_{4}\left(\theta, a_{1}\right)$.

Then let $\lambda_{3}<\lambda_{2}$. One can prove that the second term in the right-hand side in (3.8) is bounded by $\exp \left(-\frac{\lambda_{3}}{2} N \varepsilon^{2}\right)$ provided

$$
R^{2} \geq A \max \left(1, \varepsilon^{2}, \ln \left(\varepsilon^{-2}\right)\right), \quad N \varepsilon^{2} \geq B 3^{C\left(R \varepsilon^{-1}\right)^{1 / \alpha}}
$$

for positive constants $A, B$ and $C$ depending also on $\lambda_{3}$. Moreover, for $\theta=\frac{\varepsilon^{2-p} \lambda_{3}}{2 K_{3}}$, also the first term in the right-hand side in (3.8) is bounded by $\exp \left(-\frac{\lambda_{3}}{2} N \varepsilon^{2}\right)$ as soon as $R^{2} \geq R_{4} \max \left(1, \ln \left(\varepsilon^{-2}\right)\right)$, for a constant $R_{4}$ depending on $\lambda_{3}$.

Letting $R=\varepsilon\left(\frac{1}{C \ln 3} \ln \frac{N \varepsilon^{2}}{B}\right)^{\alpha}$ if $\varepsilon \in(0,1)$ and $R=\sqrt{A} \varepsilon$ otherwise, and $\alpha^{\prime}<\alpha$, both conditions in (3.9) hold true as soon as $N \geq N_{0} \varepsilon^{-2} \exp \left(N_{0} \varepsilon^{-1 / \alpha^{\prime}}\right)$ for a constant $N_{0}$ depending on $E, a, \lambda, \lambda_{3}, \alpha$ and $\alpha^{\prime}$. Finally, given $\lambda^{\prime}<\lambda_{3}<\lambda$, this condition ensures that

$$
\mathbb{P}\left[W_{p,[0, T]}\left(\mu, \hat{\mu}^{N}\right)>\varepsilon\right] \leq 2 \exp \left(-\frac{\lambda_{3}}{2} N \varepsilon^{2}\right) \leq \exp \left(-\frac{\lambda^{\prime}}{2} N \varepsilon^{2}\right),
$$

for a possibly larger $N_{0}$. This concludes the argument in the case when $p \in[1,2)$.

In the case when $p=2$, given $0<\eta<1, \lambda_{3}<\lambda_{2}$ and $a_{2}<a_{1}$, the same condition on $N$ and $\varepsilon$ (for some $N_{0}$ ) is sufficient for the bound

$$
\mathbb{P}\left[W_{2,[0, T]}\left(\mu, \hat{\mu}^{N}\right)>\varepsilon\right] \leq \exp \left(-\frac{\lambda_{3}}{2} \eta^{2} N \varepsilon^{2}\right)+\exp \left(-\frac{a_{2}}{2}(1-\eta)^{2} N \varepsilon^{2}\right)
$$


to hold (by (3.4)). One optimizes this bound by letting

$$
a_{2}=a \frac{\lambda_{3}}{\lambda}(\in(0, a)) \text { and } \quad \eta=\frac{\sqrt{a_{2}}}{\sqrt{a_{2}}+\sqrt{\lambda_{3}}}
$$

Given $\lambda^{\prime}<\lambda_{3}<\lambda$, this ensures the existence of $N_{0}$ such that

$$
\mathbb{P}\left[W_{2,[0, T]}\left(\mu, \hat{\mu}^{N}\right)>\varepsilon\right] \leq 2 \exp \left(-\frac{\lambda_{3}}{2} \frac{a}{(\sqrt{a}+\sqrt{\lambda})^{2}} N \varepsilon^{2}\right) \leq \exp \left(-\frac{\lambda^{\prime}}{2} \frac{1}{(1+\sqrt{\lambda / a})^{2}} N \varepsilon^{2}\right) .
$$

for any $\varepsilon>0$ and $N \geq N_{0} \varepsilon^{-2} \exp \left(N_{0} \varepsilon^{-1 / \alpha^{\prime}}\right)$. This concludes the proof of Proposition 3.1 in this second and last case.

\section{INTEGRABILITY IN HÖLDER NORM}

In Section 2 we have reduced the issue of measuring the distance between $\mu_{[0, T]}$ and $\hat{\mu}_{[0, T]}^{N}$ to measuring the distance between $\mu_{[0, T]}$ and the empirical measure $\hat{\nu}_{[0, T]}^{N}$ of $N$ independent random variables drawn according to $\mu_{[0, T]}$.

We now solve the latter issue by proving that the measure $\mu_{[0, T]}$ fulfills the hypotheses of Proposition 3.1 with $p=1$, namely, that there exist $\alpha \in(0,1]$ and $a>0$ such that

$$
\mathbb{E} \exp \left(a\|X\|_{\alpha}^{2}\right):=\int_{\mathcal{C}} \mathrm{e}^{a\|x\|_{\alpha}^{2}} \mathrm{~d} \mu_{[0, T]}(x)<+\infty .
$$

Proposition 4.1. Let $\mu_{0}$ be a probability measure on $\mathbb{R}^{d}$ with a finite square exponential moment and let $X_{0}$ be with law $\mu_{0}$. Given $T \geq 0$, let $X$ be the solution on $[0, T]$ to $(1.1)$ starting at $X_{0}$, where $b$ and c are Lipschitz on $\mathbb{R}^{d}$. Then, for any $\alpha \in(0,1 / 2)$, there exists $a>0$, depending on $\mu_{0}$ only through a finite square exponential moment, such that $\mathbb{E} \exp \left(a\|X\|_{\alpha}^{2}\right)$ be finite.

Proof. Assuming this result for the moment we can now conclude the proof of Theorem 1.1. Let indeed $\alpha$ be given in $(0,1 / 2)$ and $\alpha_{0} \in(\alpha, 1 / 2)$. Then, by Propositions 3.1 and 4.1 , applied with $\alpha=\alpha_{0}$ and $\alpha^{\prime}=\alpha$, there exist positive constants $\tilde{K}$ and $\tilde{N}_{0}$, depending on $\alpha_{0}, \alpha, T$ and a square exponential moment of $\mu_{0}$, such that

$$
\mathbb{P}\left[W_{1,[0, T]}\left(\mu_{[0, T]}, \hat{\nu}_{[0, T]}^{N}\right)>\tilde{\varepsilon}\right] \leq \mathrm{e}^{-\tilde{K} N \tilde{\varepsilon}^{2}}
$$

for any $\tilde{\varepsilon}>0$ and $N \geq \tilde{N}_{0} \tilde{\varepsilon}^{-2} \exp \left(\tilde{N}_{0} \tilde{\varepsilon}^{-1 / \alpha}\right)$, where $\hat{\nu}_{[0, T]}^{N}$ is defined by (1.9) and (2.1). Then, by Proposition 2.1, there exist some constants $C$, depending only on $T$, and then $K$ and $N_{0}$, depending on $\alpha_{0}, \alpha, T$ and a finite square exponential moment of $\mu_{0}$, such that

$$
\mathbb{P}\left[W_{1,[0, T]}\left(\mu_{[0, T]}, \hat{\mu}_{[0, T]}^{N}\right)>\varepsilon\right] \leq \mathbb{P}\left[W_{1,[0, T]}\left(\mu_{[0, T]}, \hat{\nu}_{[0, T]}\right)>\varepsilon / C\right] \leq \mathrm{e}^{-K N \varepsilon^{2}}
$$

for any $\varepsilon>0$ and $N \geq N_{0} \varepsilon^{-2} \exp \left(N_{0} \varepsilon^{-1 / \alpha}\right)$. This concludes the argument.

Proof of Proposition 4.1. We separately prove the existence of positive constants $a_{1}$ and $a_{2}$ such that $\mathbb{E} \exp \left(a_{1}\|X\|_{\infty}^{2}\right)$ and $\mathbb{E} \exp \left(a_{2}[X]_{\alpha}^{2}\right)$ be finite. We only sketch the proof for the uniform norm and give it in detail for the Hölder seminorm, refering to [4] for further details.

1. For the expectation in uniform norm:

1.1. we first prove that $\mathbb{E}\left|X_{t}\right|^{2}$ is bounded on $[0, T]$ by applying Itô's formula to $\left(\left|X_{t}\right|^{2}\right)_{t \geq 0}$; 
1.2. then we prove the existence of $K>0$ such that $\mathbb{E} \exp \left(K\left|X_{t}\right|^{2}\right)$ be bounded on $[0, T]$. In fact we prove the existence of a positive and decreasing function $k$ on $[0, T]$ such that $\mathbb{E} \exp \left(k(t)\left|X_{t}\right|^{2}\right)$ be finite and bounded on $[0, T]$; for that purpose we apply Itô's formula to the process $\left(\exp k(t)\left|X_{t}\right|^{2}\right)_{t \geq 0}$. Then we let $K=k(T)>0$;

1.3. we finally use Doob's inequalities to obtain the existence of $a_{1}>0$, depending on $\mu_{0}$ only through a square-exponential moment, such that $\mathbb{E} \exp \left(a_{1}\|X\|_{\infty}^{2}\right)$ be finite.

2. We now turn to the expectation in Hölder seminorm. We first write the solution as

$$
X_{t}=X_{0}+\sigma B_{t}-\int_{0}^{t}\left(b\left(X_{s}\right)+c * \mu_{s}\left(X_{s}\right)\right) \mathrm{d} s
$$

so that

$$
[X]_{\alpha} \leq[\sigma B]_{\alpha}+\left[\int_{0}^{\cdot}\left(b\left(X_{s}\right)+c * \mu_{s}\left(X_{s}\right)\right) \mathrm{d} s\right]_{\alpha}
$$

almost surely; here $\int_{0}^{\cdot} \varphi(s) \mathrm{d} s$ is an antiderivative of $\varphi$. Hence, by Cauchy-Schwarz' inequality,

$$
\mathbb{E} \exp \left(a_{2}[X]_{\alpha}^{2}\right) \leq\left(\mathbb{E} \exp \left(4 a_{2}\|\sigma\|^{2}[B]_{\alpha}^{2}\right)\right)^{1 / 2}\left(\mathbb{E} \exp 4 a_{2}\left[\int_{0}^{\cdot}\left(b\left(X_{s}\right)+c * \mu_{s}\left(X_{s}\right)\right) \mathrm{d} s\right]_{\alpha}^{2}\right)^{1 / 2}
$$

But $\mathbb{E} \exp \left(4 a_{2}\|\sigma\|^{2}[B]_{\alpha}^{2}\right)$ is finite for $a_{2}$ small enough (see [12], Thm. 1.3.2 for instance). On the other hand, by step 1.1 and assumption on $b$ and $c$, there is a constant $A$ such that

$$
\left|b(x)+c * \mu_{s}(x)\right| \leq A+(B+\Gamma)|x|
$$

for all $x \in \mathbb{R}^{d}$ and $s \in[0, T]$; here $B$ and $\Gamma$ are the Lipschitz seminorms of $b$ and $c$. In particular

$$
\left[\int_{0}^{\cdot} b\left(X_{s}\right)+c * \mu_{s}\left(X_{s}\right) \mathrm{d} s\right]_{\alpha} \leq \sup _{0 \leq s<t \leq T} \frac{1}{|t-s|^{\alpha}} \int_{s}^{t} A+(B+\Gamma)\left|X_{u}\right| \mathrm{d} u \leq T^{1-\alpha}\left(A+(B+\Gamma)\|X\|_{\infty}\right)
$$

almost surely, and

$$
\mathbb{E} \exp 4 a_{2}\left[\int_{0}^{\cdot}\left(b\left(X_{s}\right)+c * \mu_{s}\left(X_{s}\right)\right) \mathrm{d} s\right]_{\alpha}^{2} \leq \exp \left(8 a_{2} T^{2-2 \alpha} A^{2}\right) \mathbb{E} \exp \left(8 a_{2} T^{2-2 \alpha}(B+\Gamma)^{2}\|X\|_{\infty}^{2}\right)
$$

which by step 1 is finite as soon as $8 a_{2} T^{2-2 \alpha}(B+\Gamma)^{2} \leq a_{1}$.

On the whole, $\mathbb{E} \exp \left(a_{2}[X]_{\alpha}^{2}\right)$ is indeed finite for $a_{2}$ small enough, depending on $\mu_{0}$ only through a finite square exponential moment, which concludes the argument.

\section{An eXAmple of application}

In this section we give an instance of error bound in the approximation by $\frac{1}{N} \sum_{i=1}^{N} \varphi\left(X^{i}\right)$ of the expectation of a quantity $\varphi(X)$ depending on the whole path of the considered process. 
Let $0<t_{1}<\cdots<t_{n} \leq T$ and for $1 \leq j \leq n$ let $B_{j}$ be the (for instance closed) ball $B\left(x_{j}, r_{j}\right)$ of center $x_{j}$ and radius $r_{j}>0$ in the Euclidean space $\mathbb{R}^{d}$. Then we can approximate the probability $\mathbb{P}\left[X_{t_{j}} \in B_{j} ; 1 \leq j \leq n\right]$ for $X_{t}$ to be in $B_{j}$ at $t=t_{j}$ for each $j$ as follows:

In the notation and assumptions of Theorem 1.1, assume moreover that all partial derivatives $\partial^{m} b$ and $\partial^{m} c$ of $b$ and $c$ are continuous and bounded on $\mathbb{R}^{d}$, for any multi-index $m \in \mathbb{N}^{d}$ with $1 \leq|m| \leq s$ where $s$ is the smallest integer number larger than $d / 2$, and that the diffusion matrix $\sigma$ is (for instance) a nonzero multiple of the identity, as in the example (1.4). Then, for any $\alpha \in(0,1 / 2)$, there exist positive constants $K$ and $N_{0}$ such that

$$
\mathbb{P}\left[\left|\frac{1}{N} \sum_{i=1}^{N} \prod_{j=1}^{n} \chi_{j}\left(X_{t_{j}}^{i}\right)-\mathbb{P}\left[X_{t_{j}} \in B_{j} ; 1 \leq j \leq n\right]\right|>\varepsilon\right] \leq \mathrm{e}^{-K N \varepsilon^{4}}
$$

for all $\varepsilon \in(0,1)$ and $N \geq N_{0} \varepsilon^{-4} \exp \left(N_{0} \varepsilon^{-2 / \alpha}\right)$.

Here $\chi_{j}$ is the indicator function of the ball $B_{j}$ defined by $\chi_{j}(x)=1$ if $x \in B_{j}$ and 0 otherwise, and let us note that only the $\varepsilon \in(0,1)$ have to be considered.

Indeed

$$
\begin{array}{r}
\mid \frac{1}{N} \sum_{i=1}^{N} \prod_{j=1}^{n} \chi_{j}\left(X_{t_{j}}^{i}\right) \\
-\mathbb{P}\left[X_{t_{j}} \in B_{j} ; 1 \leq j \leq n\right]|=| \int_{\mathcal{C}} \prod_{j=1}^{n} \chi_{j}\left(f\left(t_{j}\right)\right) \mathrm{d} \hat{\mu}^{N}(f)-\int_{\mathcal{C}} \prod_{j=1}^{n} \chi_{j}\left(f\left(t_{j}\right)\right) \mathrm{d} \mu(f) \mid \\
\leq \int_{\mathcal{C}}\left|\prod_{j=1}^{n} \chi_{j}\left(f\left(t_{j}\right)\right)-\prod_{j=1}^{n} \varphi_{j}\left(f\left(t_{j}\right)\right)\right| \mathrm{d} \hat{\mu}^{N}(f)+\left|\int_{\mathcal{C}} \prod_{j=1}^{n} \varphi_{j}\left(f\left(t_{j}\right)\right) \mathrm{d}\left(\hat{\mu}^{N}-\mu\right)(f)\right| \\
+\int_{\mathcal{C}}\left|\prod_{j=1}^{n} \varphi_{j}\left(f\left(t_{j}\right)\right)-\prod_{j=1}^{n} \chi_{j}\left(f\left(t_{j}\right)\right)\right| \mathrm{d} \mu(f) .
\end{array}
$$

Here $\varphi_{j}$ is the $\mathbb{R}^{d}$ to $\mathbb{R}$ map defined by $\varphi_{j}(x)=\left(1-\frac{\mathrm{d}\left(x, B_{j}\right)}{\delta}\right)_{+}$for some $0<\delta \leq \min _{1 \leq j \leq n} r_{j}$ to be chosen later, where $\mathrm{d}(x, A)$ is the distance of a point $x$ to a set $A$ and $u_{+}=\max (u, 0)$ for all real $u$. For simplicity we write $\hat{\mu}^{N}, \mu$ and $W_{1}$ instead of $\hat{\mu}_{[0, T]}^{N}, \mu_{[0, T]}$ and $W_{1,[0, T]}$ respectively.

The second term in (5.1), which is the main term, will be bounded by the Kantorovich-Rubinstein formulation (1.7). Indeed, if $f$ and $g$ are two functions in $\mathcal{C}$, then

$$
\left|\prod_{j=1}^{n} \varphi_{j}\left(f\left(t_{j}\right)\right)-\prod_{j=1}^{n} \varphi_{j}\left(g\left(t_{j}\right)\right)\right| \leq \sum_{j=1}^{n}\left|\varphi_{j}\left(f\left(t_{j}\right)\right)-\varphi_{j}\left(g\left(t_{j}\right)\right)\right|
$$

since the maps $\varphi_{j}$ take values in $[0,1]$ and because of the following elementary bound:

Lemma 5.1. If $a_{j}$ and $b_{j}$ are real numbers in $[0,1]$ for $1 \leq j \leq n$, then

$$
\left|\prod_{j=1}^{n} a_{j}-\prod_{j=1}^{n} b_{j}\right| \leq \sum_{j=1}^{n}\left|a_{j}-b_{j}\right|
$$

Then the maps $\varphi_{j}$ are $\delta^{-1}$-Lipschitz, so

$$
\sum_{j=1}^{n}\left|\varphi_{j}\left(f\left(t_{j}\right)\right)-\varphi_{j}\left(g\left(t_{j}\right)\right)\right| \leq n \delta^{-1}\|f-g\|_{\infty}
$$


Consequently the map $f \mapsto \prod_{j=1}^{n} \varphi_{j}\left(f\left(t_{j}\right)\right)$ is $n \delta^{-1}$-Lipschitz on $\mathcal{C}$, and by $(1.7)$ the second term in $(5.1)$ is bounded by $n \delta^{-1} W_{1}\left(\hat{\mu}^{N}, \mu\right)$.

Then the first term in (5.1) is bounded by

$$
\int_{\mathcal{C}} \sum_{j=1}^{n}\left|\chi_{j}\left(f\left(t_{j}\right)\right)-\varphi_{j}\left(f\left(t_{j}\right)\right)\right| \mathrm{d} \hat{\mu}^{N}(f)
$$

by Lemma 5.1 again. Now, for all $1 \leq j \leq n$ and $x \in \mathbb{R}^{d}$,

$$
\left|\chi_{j}(x)-\varphi_{j}(x)\right|=\varphi_{j}(x)-\chi_{j}(x) \leq \gamma_{j}(x)
$$

where $\gamma_{j}(x)=\left(1-\frac{\mathrm{d}\left(x, B_{j}\right)+\mathrm{d}\left(x, \mathbb{R}^{d} \backslash B_{j}\right)}{\delta}\right)_{+}$. The map $x \mapsto \mathrm{d}\left(x, B_{j}\right)+\mathrm{d}\left(x, \mathbb{R}^{d} \backslash B_{j}\right)$ is 1 -Lipschitz, so $\gamma_{j}$ is $\delta^{-1}$-Lipschitz, and by (1.7) again the first term in (5.1) is bounded by

$$
\sum_{j=1}^{n} \int_{\mathcal{C}} \gamma_{j}\left(f\left(t_{j}\right)\right) \mathrm{d} \hat{\mu}^{N}(f) \leq \sum_{j=1}^{n}\left[\delta^{-1} W_{1}\left(\hat{\mu}^{N}, \mu\right)+\int_{\mathcal{C}} \gamma_{j}\left(f\left(t_{j}\right)\right) \mathrm{d} \mu(f)\right] .
$$

Moreover, if for any $j$ we let $\mu_{t_{j}}$ be the marginal at time $t_{j}$ of the distribution $\mu$, then

$$
\int_{\mathcal{C}} \gamma_{j}\left(f\left(t_{j}\right)\right) \mathrm{d} \mu(f)=\int_{\mathbb{R}^{d}} \gamma_{j}(x) \mathrm{d} \mu_{t_{j}}(x) \leq \mu_{t_{j}}\left[C_{j}\right]
$$

where $C_{j}=B\left(x_{j}, r_{j}+\delta\right) \backslash B\left(x_{j}, r_{j}-\delta\right)$, since $\gamma_{j}$ is zero outside of $C_{j}$ and bounded by 1 on $C_{j}$.

In the same way the third term in (5.1) is bounded by

$$
\sum_{j=1}^{n} \mu_{t_{j}}\left[D_{j}\right]
$$

where $D_{j}=B\left(x_{j}, r_{j}+\delta\right) \backslash B\left(x_{j}, r_{j}\right)$, since $\varphi_{j}-\chi_{j}$ is zero outside of $D_{j}$ and bounded by 1 on $D_{j}$.

Now, under our assumptions, we can adapt the techniques in [6] Theorem B.1 to prove that for any $t>0$ the time marginal $\mu_{t}$ belongs to the Sobolev space $H^{s}\left(\mathbb{R}^{d}\right)$, with $s>d / 2$, whence to $L^{\infty}\left(\mathbb{R}^{d}\right)$. Moreover there exists a constant $K_{1}$, depending only on $t_{1}, t_{n}, d, b, c, \sigma$ and a square exponential moment of $\mu_{0}$, such that

$$
\sup _{t_{1} \leq t \leq t_{n}}\left\|\mu_{t}\right\|_{L^{\infty}} \leq K_{1}
$$

In particular the first and third terms in (5.1) are together bounded by

$$
n \delta^{-1} W_{1}\left(\hat{\mu}^{N}, \mu\right)+K_{1} \sum_{j=1}^{n} \operatorname{Leb}\left[C_{j}\right]+\operatorname{Leb}\left[D_{j}\right]
$$

where Leb $[A]$ stands for the Lebesgue measure of a Borel set $A$ in $\mathbb{R}^{d}$.

Moreover

$$
\operatorname{Leb}\left[C_{j}\right]=\omega_{d}\left(\left(r_{j}+\delta\right)^{d}-\left(r_{j}-\delta\right)^{d}\right) \leq 2^{d} \omega_{d} r_{j}^{d-1} \delta
$$


and

$$
\operatorname{Leb}\left[D_{j}\right]=\omega_{d}\left(\left(r_{j}+\delta\right)^{d}-r_{j}^{d}\right) \leq\left(2^{d}-1\right) \omega_{d} r_{j}^{d-1} \delta
$$

for any $1 \leq j \leq n$ and $0<\delta \leq r_{j}$, where $\omega_{d}$ is the Lebesgue measure of the Euclidean unit ball in $\mathbb{R}^{d}$. Hence it follows from (5.1) that for a constant $K_{2}$, independent of $N$ and the $r_{j}$,

$$
\left|\frac{1}{N} \sum_{i=1}^{N} \prod_{j=1}^{n} \chi_{j}\left(X_{t_{j}}^{i}\right)-\mathbb{P}\left[X_{t_{j}} \in B_{j} ; 1 \leq j \leq n\right]\right| \leq 2 n \delta^{-1} W_{1}\left(\hat{\mu}^{N}, \mu\right)+K_{2} \sum_{j=1}^{n} r_{j}^{d-1} \delta
$$

for any $0<\delta \leq \min _{1 \leq j \leq n} r_{j}$ and in particular

$$
\mathbb{P}\left[\left|\frac{1}{N} \sum_{i=1}^{N} \prod_{j=1}^{n} \chi_{j}\left(X_{t_{j}}^{i}\right)-\mathbb{P}\left[X_{t_{j}} \in B_{j} ; 1 \leq j \leq n\right]\right|>\varepsilon\right] \leq \mathbb{P}\left[W_{1}\left(\hat{\mu}^{N}, \mu\right)>\frac{\delta}{2 n}\left(\varepsilon-K_{2} R \delta\right)\right]
$$

for any $0<\delta \leq r$, in the notation $r=\min _{1 \leq j \leq n} r_{j}$ and $R=\sum_{j=1}^{n} r_{j}^{d-1}$.

In the case when $\varepsilon \leq 2 K_{2} R r$ we choose $\delta=\frac{\varepsilon}{2 K_{2} R} \in(0, r]$, so that, by Theorem 1.1, for any $\alpha \in(0,1 / 2)$ there exist some constants $K$ and $N_{0}$, independent of $\varepsilon$ and $N$, such that

$$
\mathbb{P}\left[W_{1}\left(\hat{\mu}^{N}, \mu\right)>\frac{\delta}{2 n}\left(\varepsilon-K_{2} R \delta\right)\right]=\mathbb{P}\left[W_{1}\left(\hat{\mu}^{N}, \mu\right)>\frac{\varepsilon^{2}}{8 K_{2} R n}\right] \leq \exp \left(-K N\left(\frac{\varepsilon^{2}}{8 K_{2} R n}\right)^{2}\right)
$$

for all $N \geq N_{0}\left(\frac{\varepsilon^{2}}{8 K_{2} R n}\right)^{-2} \exp \left(N_{0}\left(\frac{\varepsilon^{2}}{8 K_{2} R n}\right)^{-1 / \alpha}\right)$.

In the case when $\varepsilon>2 K_{2} R r$ we choose $\delta=r$, so that

$$
\mathbb{P}\left[W_{1}\left(\hat{\mu}^{N}, \mu\right)>\frac{\delta}{2 n}\left(\varepsilon-K_{2} R \delta\right)\right] \leq \mathbb{P}\left[W_{1}\left(\hat{\mu}^{N}, \mu\right)>\frac{\varepsilon r}{4 n}\right] \leq \exp \left(-K N\left(\frac{\varepsilon r}{4 n}\right)^{2}\right)
$$

for all $N \geq N_{0}\left(\frac{\varepsilon r}{4 n}\right)^{-2} \exp \left(N_{0}\left(\frac{\varepsilon r}{4 n}\right)^{-1 / \alpha}\right)$.

As a conclusion, there exist two new constants $K$ and $N_{0}$, depending on $\mu_{0}$ only through a square exponential moment, such that

$$
\mathbb{P}\left[\left|\frac{1}{N} \sum_{i=1}^{N} \prod_{j=1}^{n} \chi_{j}\left(X_{t_{j}}^{i}\right)-\mathbb{P}\left[X_{t_{j}} \in B_{j} ; 1 \leq j \leq n\right]\right|>\varepsilon\right] \leq \mathrm{e}^{-K N \varepsilon^{4}}
$$

for all $\varepsilon \in(0,1)$ and $N \geq N_{0} \varepsilon^{-4} \exp \left(N_{0} \varepsilon^{-2 / \alpha}\right)$. This concludes the argument.

Acknowledgements. The author thanks Professor M. Ledoux for his kind interest in this work, in particular pointing out reference [12], and Professors A. Guillin and C. Villani for stimulating discussions during the preparation of [6]. 


\section{REFERENCES}

[1] S. Benachour, B. Roynette, D. Talay and P. Vallois, Nonlinear self-stabilizing processes. I: Existence, invariant probability, propagation of chaos. Stoch. Proc. Appl. 75 (1998) 173-201.

[2] D. Benedetto, E. Caglioti, J.A. Carrillo and M. Pulvirenti, A non-Maxwellian steady distribution for one-dimensional granular media. J. Statist. Phys. 91 (1998) 979-990.

[3] S. Bobkov and F. Götze, Exponential integrability and transportation cost related to logarithmic Sobolev inequalities. J. Funct. Anal. 163 (1999) 1-28.

[4] F. Bolley, Quantitative concentration inequalities on sample path space for mean field interaction. Available online at www. ceremade.dauphine.fr/ bolley (2008).

[5] F. Bolley and C. Villani, Weighted Csiszár-Kullback-Pinsker inequalities and applications to transportation inequalities. Ann. Fac. Sci. Toulouse Math. 6 (2005) 331-352.

[6] F. Bolley, A. Guillin and C. Villani, Quantitative concentration inequalities for empirical measures on non-compact spaces. Probab. Theory Relat. Fields 137 (2007) 541-593.

[7] J.A. Carrillo, R.J. McCann and C. Villani, Contractions in the 2-Wasserstein length space and thermalization of granular media. Arch. Rat. Mech. Anal. 179 (2006) 217-263.

[8] P. Cattiaux, A. Guillin and F. Malrieu, Probabilistic approach for granular media equations in the non uniformly case. Probab. Theory Relat. Fields 140 (2008) 19-40.

[9] A. Dembo and O. Zeitouni, Large deviations techniques and applications. Springer, NewYork (1998).

[10] H. Djellout, A. Guillin and L. Wu, Transportation cost-information inequalities and applications to random dynamical systems and diffusions. Ann. Probab. 32 (2004) 2702-2732.

[11] J. Dolbeault, Free energy and solutions of the Vlasov-Poisson-Fokker-Planck system: external potential and confinement (large time behavior and steady states). J. Math. Pures Appl. 9 (1999) 121-157.

[12] X. Fernique, Régularité des trajectoires des fonctions aléatoires gaussiennes. Lect. Notes Math. 480. Springer, Berlin (1975).

[13] N. Gozlan, Principe conditionnel de Gibbs pour des contraintes fines approchées et inégalités de transport. Thèse de doctorat de l'Université de Paris 10-Nanterre, 2005).

[14] S.R. Kulkarni and O. Zeitouni, A general classification rule for probability measures. Ann. Statist. 23 (1995) $1393-1407$.

[15] G.G. Lorentz, Approximation of functions. Holt, Rinehart and Winston, New York (1966).

[16] F. Malrieu, Logarithmic Sobolev inequalities for some nonlinear PDE's. Stoch. Proc. Appl. 95 (2001) 109-132.

[17] S. Méléard, Asymptotic behaviour of some interacting particle systems; McKean-Vlasov and Boltzmann models. Lect. Notes Math. 1627. Springer, Berlin (1996).

[18] A.-S. Sznitman, Topics in propagation of chaos. Lect. Notes Math. 1464. Springer, Berlin (1991).

[19] A. van der Vaart and J. Wellner, Weak convergence and empirical processes. Springer, Berlin (1995).

[20] C. Villani, Topics in optimal transportation, volume 58 of Grad. Stud. Math. A.M.S., Providence (2003). 